\title{
Generalized AdS/CFT Dualities and Space-Time Symmetries of M/Superstring Theory
}

\author{
Murat Günaydin'
}

Penn State University

Physics Department

University Park, PA 16802

\begin{abstract}
I review the relationship between AdS/CFT ( anti-de Sitter / conformal field theory) dualities and the general theory of unitary lowest weight (ULWR) (positive energy) representations of non-compact space-time groups and supergroups. The ULWR's have the remarkable property that they can be constructed by tensoring some fundamental ULWR's ( singletons or doubletons). Furthermore, one can go from the manifestly unitary compact basis of the ULWR's of the conformal group (Wigner picture) to the manifestly covariant coherent state basis ( Dirac picture) labelled by the space-time coordinates. Hence every irreducible ULWR corresponds to a covariant field with a definite conformal dimension. These results extend to higher dimensional generalized spacetimes ( superspaces) defined by Jordan (super) algebras and Jordan (super) triple systems. In particular, they extend to the ULWR's of the M-theory symmetry superalgebra $O S p(1 / 32, \mathbf{R})$.
\end{abstract}

Invited talk given at the IXth Marcel Grossmann Meeting in Rome, July, 2000

\footnotetext{
${ }^{1}$ Work supported in part by the National Science Foundation under Grant Number PHY-9802510.

e-mail: murat@phys.psu.edu
} 


\section{Introduction}

The conjecture of Maldacena [1, 2, 3, 4] on the duality between the large $\mathcal{N}$ limits of certain conformal field theories (CFT) in $d$ dimensions and the superstring theory on the products of $d+1$ dimensional anti-de Sitter (AdS) spaces with spheres fits perfectly into the general theory of the construction of positive energy representations of AdS and conformal groups and supergroups by a simple tensoring procedure from some "fundamental" representations as reviewed in [5, 6]. These "fundamental" representations have been called singletons or doubletons and act like the basic building blocks (quarks) of all the positive energy representations. For simple noncompact groups including AdS and conformal groups the general theory was developed in [7] . The extension of the general theory to noncompact supergroups including AdS and conformal supergroups was given in [8].

The supermultiplets of fields of the superconformal field theories to which the superstring/M-theory is dual to in various dimensions are precisely the "CPT self-conjugate" singleton or doubleton supermultiplets of the corresponding space-time supergroups.

This simple tensoring procedure was applied to the construction of the Kaluza-Klein spectra of ten and eleven dimensional supergravity theories long time ago. In [9] the spectrum of the $S^{7}$ compactification of eleven dimensional supergravity was shown to fit into an infinite tower of short unitary supermultiplets of $O S p(8 / 4, R)$. The ultra-short singleton supermultiplet of $O S p(8 / 4, R)$ sits at the bottom of this infinite tower of Kaluza-Klein modes and decouple from the spectrum as local gauge degrees of freedom [9]. However, even though it decouples from the spectrum as local gauge modes, one can generate the entire spectrum of 11-dimensional supergravity over $S^{7}$ by tensoring $p$ copies ("colors") $(p=2,3,4, \ldots)$ of singleton supermultiplets and restricting oneself to "CPT self-conjugate" supermultiplets.

The spectrum of 11-d supergravity over the four sphere $S^{4}$ 10, 11 was shown to fit into an infinite tower of unitary supermultiplets of $O S p\left(8^{*} / 4\right)$ with the even subgroup $S O(6,2) \times U S p(4)$ in [10]. Again the vacuum doubleton supermultiplet of $O S p\left(8^{*} / 4\right)$ decouples from the spectrum as local gauge degrees of freedom and the entire physical spectrum of 11-dimensional supergravity over $S^{4}$ was obtained by simply tensoring an arbitrary number (colors) of these doubleton supermultiplets and restricting oneself to the vacuum ("CPT self-conjugate) supermultiplets [10]. 
The spectrum of the $S^{5}$ compactification of ten dimensional IIB supergravity was calculated in [12, 13]. The entire spectrum falls into an infinite tower of massless and massive unitary supermultiplets of $N=8 A d S_{5}$ superalgebra $S U(2,2 / 4)$ [12]. The "CPT self-conjugate" doubleton supermultiplet of $N=8 A d S$ superalgebra decouples from the physical spectrum as local gauge degrees of freedom. By tensoring it with itself repeatedly and restricting oneself to the $C P T$ self-conjugate vacuum supermultiplets one generates the entire spectrum of Kaluza-Klein states of ten dimensional IIB supergravity on $S^{5}$.

As was pointed out in [12, 14] the CPT self-conjugate $N=8 A d S_{5}$ doubleton supermultiplet does not have a Poincaré limit in five dimensions and its field theory is the conformally invariant $N=4$ super Yang-Mills theory in $d=4$ Minkowski space which can be identified as the boundary of $A d S_{5}$.

Similarly, the singleton supermultiplet of $\operatorname{SSp}(8 / 4, R)$ and the doubleton supermultiplet of $O S p\left(8^{*} / 4\right)$ do not have a Poincaré limit in $d=4$ and $d=7$, respectively, and their field theories are conformally invariant theories in one lower dimension 2. Thus we see that at the level of physical states the proposal of Maldacena is perfectly consistent with the above mentioned results.

\section{Massless and Massive Supermultiplets of Anti-de Sitter Supergroups}

The Poincaré limit of the remarkable representations (singletons) of the $d=4 A d S$ group $S O(3,2)$ discovered by Dirac [15] are known to be singular [16]. However, the tensor product of two singleton representations decomposes into an infinite set of massless unitary irreducible representations which do have a smooth Poincaré limit [16, 17, 17]. Similarly, the tensor product of two singleton supermultiplets of $N$ extended $A d S_{4}$ supergroup $O S p(N / 4, R)$ decomposes into an infinite set of massless supermultiplets which do have a Poincaré limit in $A d S_{5}$ [17, 9, 18, 19]. In contrast to $A d S_{4}$ group $S O(3,2)$, the $A d S_{5}$ group $S U(2,2)$ and $A d S_{7}$ group $S O(6,2)$ admit infinitely many "remarkable" representations that do not have a Poincaré limit in 5 and 7 di-

\footnotetext{
${ }^{2}$ see [5] for references
} 
mensions , respectively. These representations have been called " doubletons" since they require two sets of oscillators for their realization [10, 12]. The doubleton supermultiplets of extended $A d S$ supergroups in $d=5(S U(2,2 / N))$ and $d=7\left(O S p\left(8^{*} / 2 N\right)\right)$ share the same remarkable features of the singleton supermultiplets of $d=4 A d S$ supergroups i.e the tensor product of any two doubletons decompose into an infinite set of massless supermultiplets [10, 12, 19, 20, 21, 22]. In $d=3$ the $A d S$ group $S O(2,2)$ is not simple and is isomorphic to $S O(2,1) \times S O(2,1)$. Each $S O(2,1)$ factor can be extended to a simple superalgebra with some internal symmetry group and one has a rich variety of $A d S$ supergroups in $d=3$ whose unitary supermultiplets were studied in 23.

The tensor product of more than two copies of the singleton or doubleton supermultiplets of $A d S$ supergroups decompose into an infinite set of massive supermultiplets [10, 9, 12, 6, 20, 21, 22, 24, 25, 26, 27] . The KaluzaKlein spectrum of the 11-dimensional and ten dimensional IIB supergravity compactified over $S^{7}\left(S^{4}\right)$ and $S^{5}$ fall into CPT self-conjugate short massless and massive supermultiplets of $O S p(8 \mid 4, R)\left(O S p\left(8^{*} \mid 4\right)\right)$ and $S U(2,2 \mid 4)$, respectively. As explained above they can all be obtained by tensoring $p$ copies $(p=1,2,3, \ldots)$ of the corresponding singleton or doubleton supermultiplets and restricting oneself to CPT-self conjugate irreducible multiplets. If one does not restrict oneself to self-conjugate supermultiplets then tensoring procedure yields intermediate as well as long massive supermultiplets of the corresponding supergroups. These intermediate and long supermultiplets are expected to descend from the massive modes of M/Superstring theory [20, 21, 27].

\section{Generalized Space-times}

\subsection{Rotation, Lorentz and Conformal Groups of Gen- eralized Spacetimes}

In the twistor formalism a coordinate vector $x_{\mu}$ in four-dimensional spacetime is naturally represented as a $2 \times 2$ Hermitian matrix $x=x_{\mu} \sigma^{\mu}$ over the field of complex numbers $\mathbf{C}$. Since the Hermitian matrices over the field of complex numbers close under the symmetric anti-commutator product one can thus regard the coordinate vectors as elements of a Jordan algebra 
denoted as $J_{2}^{\mathbf{C}}$ 28, 29. Then the rotation, Lorentz and conformal groups in $d=4$ can be identified with the automorphism, reduced structure and Möbius ( linear fractional) groups of the Jordan algebra of $J_{2}^{\mathrm{C}}$ [28, 29]. Furthermore, this interpretation allows one to define generalized space-times whose coordinates are parametrized by the elements of Jordan algebras (or Jordan triple systems) [28, 29, 30] . The rotation $\operatorname{Rot}(J), \operatorname{Lorentz} \operatorname{Lor}(J)$ and conformal $C o n(J)$ groups of these generalized space-times are then identified with the automorphism $\operatorname{Aut}(J)$, reduced structure $\operatorname{Str}_{0}(J)$ and Möbius Mö $(J)$ groups of the corresponding Jordan algebra [28, 29, 31, 30]. A complete list of the generalized spacetimes defined by formally real Jordan algebras and triple systems (JTS) as well as their symmetry groups were given in 31, 30].

\subsection{Conformal Fields over Generalized Spacetimes and the Positive Energy Unitary Representations of Conformal Groups}

Remarkably, the list of generalized conformal groups defined by simple formally real Jordan algebras and JTS's corresponds precisely to the list of simple non-compact groups that admit positive energy unitary representations (i.e unitary representations of the lowest weight type (ULWR)). Furthermore, the maximal compact subgroups of the generalized conformal groups are simply the compact forms of their structure groups (generalized Lorentz group times dilatations). For example, the conformal group of the Jordan algebra

$J_{2}^{\mathbf{C}}$ corresponding to the four dimensional Minkowski space is $S U(2,2)$ with a maximal compact subgroup $S U(2) \times S U(2) \times U(1)$ which is simply the compact form of the structure group $S L(2, \mathbf{C}) \times \mathbf{S O}(\mathbf{1}, \mathbf{1})$. In 21 it was explicitly shown how to go from the compact $S U(2) \times S U(2) \times U(1)$ basis of the ULWR's of $S U(2,2)$ to the manifestly covariant $S L(2, \mathbf{C}) \times \mathbf{S O}(\mathbf{1}, \mathbf{1})$ basis. The transition from the compact to the covariant basis corresponds simply to going from a "particle" basis to a coherent state basis of the corresponding positive energy representation. The coherent states are labelled by the elements of $J_{2}^{\mathbf{C}}$ representing the coordinates of four dimensional Minkowski space. One can then establish a one-to-one correspondence between irreducible ULWR's of $S U(2,2)$ and the conformal fields transforming irreducibly under the Lorentz group $S L(2, \mathbf{C})$ with a definite conformal dimension [21, 32]. Similarly, the 
conformal group $S O^{*}(8)$ of the Jordan algebra $J_{2}^{\mathbf{H}}$ parametrizing the six dimensional Minkowski space has a maximal compact subgroup $U(4)$ which is the compact form of the structure group $S U^{*}(4) \times S O(1,1)$. In [22] it was shown how to go from the compact $U(4)$ basis of the ULWR's of $S O^{*}(8)$ to the non-compact basis $S U^{*}(4) \times S O(1,1)$ which is simply the Lorentz group in six dimensions times dilatations. The coherent states of the non-compact basis are again labelled by the elements of the Jordan algebra $J_{2}^{\mathbf{H}}$ of $2 \times 2$ hermitian quaternionic matrices representing the coordinates of $6 \mathrm{~d}$ Minkowski space. Thus each irreducible ULWR of $S O^{*}(8)$ can be identified with a field transforming covariantly under the six dimensional Lorentz group $S U^{*}(4)$ with a definite conformal dimension. These results obtained explicitly for the conformal groups of $J_{2}^{\mathbf{C}}$ and $J_{2}^{\mathbf{H}}$ extend to the conformal groups of all formally real Jordan algebras and of Hermitian Jordan triple systems [33, 34]. Recently, the nonlinear action of these conformal groups have been generalized to novel quasiconformal group actions over certain natural extensions of the corresponding spacetimes [35].

\subsection{Generalized superspaces defined by Jordan super- algebras and the ULWR's of their symmetry su- pergroups}

The generalized space-times defined by Jordan algebras extend naturally to generalized superspaces over Jordan superalgebras and super Jordan triple systems [29, 31, 33]. One defines the generalized superspaces by multiplying the even elements of a Jordan superalgebra $J$ by real coordinates and their odd elements by Grassmann coordinates [29, 31]. The rotation, Lorentz and conformal supergroups of these generalized superspaces are then given by the automorphism, reduced structure and Möbius supergroups of $J$. A complete list of these supergroups was given in [31]. The conformal groups of formally real Jordan algebras all admit ULWR's and as explained above one can associate with each irreducible ULWR a covariant conformal field with a definite conformal dimension. The corresponding real forms of Jordan superalgebras or super Jordan triple systems are such that their conformal supergroups admit positive energy unitary representations. The general theory for the construction of the unitary lowest weight representations of non-compact supergroups was given in [8], both in a compact particle state basis as well as 
the compact super-coherent state basis. The coherent states defined in [8] for non-compact groups $G$ are labelled by the complex variables parametrizing the hermitian symmetric space $G / H$ where $H$ is the maximal compact subgroup. On the other hand the coherent states defined in 21] for $S U(2,2)$ and in [22, 36] for $O S p\left(8^{*} \mid 4\right)$ as well as their generalizations to all non-compact groups discussed in the previous section are labelled by real (generalized) coordinates of the (generalized) space-times on which $G$ acts as a (generalized) conformal group.

The irreducible ULWR's of (generalized) superconformal groups correspond simply to a supermultiplet of fields transforming irreducibly under the (generalized) Lorentz group with definite conformal dimensions. In a non-compact supercoherent state basis these unitary supermultiplets are represented by superfields [36 [3. The labels of supercoherent states are the coordinates of corresponding (generalized) superspaces.

\section{Unitary Supermultiplets of M-theory Su- peralgebra $O S p(1 \mid 32, R)$}

The simple supergroup $O S p(1 / 32, R)$ was proposed as the generalized $A d S$ supergroup in $d=11$ long time ago [38]. One can also regard $O S p(1 / 32, R)$ as the generalized conformal group in ten dimension. $\operatorname{Sp}(32, R)$ is the conformal group of the Jordan algebra $J_{16}^{\mathbf{R}}$ of $16 \times 16$ symmetric real matrices. As such one can consider $O S p(1 / 32, R)$ as the conformal supergroup of a generalized superspace of the type we discussed above. The unitary supermultiplets of $O S p(1 / 32, R)$ were studied in [6] with the aim of extending the $A d S / C F T$ duality to the maximal possible dimension in M-theory. The parity invariance of M-theory [39] requires the extension of $\operatorname{OSp}(1 / 32, R)$ to a larger supergroup that admits parity invariant representations. The "minimal" such parity symmetric supergroup is $\operatorname{OSp}(1 / 32, R)_{L} \times O S p(1 / 32, R)_{R}$ [40]. The two factors of $O S p(1 / 32, R)_{L} \times O S p(1 / 32, R)_{R}$ correspond to the embedding of left-handed and right-handed spinor representations of $S O(10,2)$ in the fundamental representation of $\operatorname{Sp}(32, R)$. The contraction to the Poincaré superalgebra with central charges proceeds via a diagonal subsupergroup

\footnotetext{
3 Recently, a number of papers studied the unitary supermultiplets of conformal supergroups in 3,4 and 6 dimensions using the formalism of superfields [37.
} 
$O S p(1 / 32, R)_{L-R}$ which contains the common subgroup $S O(10,1)$ of the two $S O(10,2)$ subgroups. The parity invariant tensor product of the singleton supermultiplets of the two factors decomposes into an infinite set of "doubleton" supermultiplets of the diagonal $\operatorname{OSp}(1 / 32, R)_{L-R}$. There is a unique "CPT self-conjugate" doubleton supermultiplet whose tensor product with itself leads to "massless" supermultiplets. The "CPT self-conjugate" massless graviton supermultiplet contains fields corresponding to those of 11-dimensional supergravity plus additional ones. One then expects the corresponding doubleton field theory to be a generalized superconformal field theory in ten dimensions that is dual to an AdS phase of M-theory in the same sense as the duality between the $N=4$ super Yang-Mills in $d=4$ and the $I I B$ superstring over $A d S_{5} \times S^{5}$. By tensoring more than two copies of the doubleton supermultiplet one obtains the massive supermultiplets of the conjectured AdS phase of M-theory.

\section{References}

[1] J. Maldacena, hep-th/9711200.

[2] E. Witten, Adv. Theor. Math. Phys. 2 (1998) 253-291, hep-th/9802150; Adv. Theor. Math. Phys. 2 (1998) 505-532, hep-th/9803131.

[3] S. S. Gubser, I. R. Klebanov and A. M. Polyakov, hep-th/9802109, Phys. Lett. B428 (1998) 105-114.

[4] O. Aharony, S. Gubser, J. Maldacena, H. Ooguri and Y. Oz, hepth/9905111.

[5] M. Günaydin and D. Minic, " Singletons, Doubletons and M-Theory", hep-th/9702047.

[6] M. Günaydin, Nucl. Phys. B528(1998) 432-450 ; hep-th/9803138.

[7] M. Günaydin and C. Saclioglu, Phys. Lett. B108 (1982) 180; Comm. Math. Phys. 87 (1982) 159.

[8] I. Bars and M. Günaydin, Comm. Math. Phys., 91 (1983) 21.

[9] M. Günaydin and N.P. Warner, Nucl. Phys. B 272 (1986) 99. 
[10] M. Günaydin, P. van Nieuwenhuizen and N.P. Warner, Nucl. Phys. B 255 (1985) 63.

[11] K. Pilch, P. K. Townsend and P. van Nieuwenhuizen, Nucl. Phys. B242 (1984) 377.

[12] M. Günaydin and N. Marcus, Class. Quantum Gravity 2 (1985) L11.

[13] H. J. Kim, L. J. Romans and P. van Nieuwenhuizen. Phys. Rev. D32 (1985) 389.

[14] M. Günaydin and N. Marcus, Class. Quant. Grav. 2 (1985) L19.

[15] P.A.M. Dirac, J. Math. Phys. 4 (1963) 901.

[16] C. Fronsdal, Phys. Rev. D 12 (1975) 3819;

M. Flato and C. Fronsdal, Lett. Math. Phys. 2 (1978) 421.

[17] M. Günaydin, Oscillator-like Unitary Representations of Non-compact Groups and Supergroups and Extended Supergravity Theories, expanded version of the invited talk given in Group Theoretical Methods in Physics, Istanbul, 1982, Ecole Normale Superieure preprint LPTENS 83/5 and in Lecture Notes in Physics Vol. 180 (1983), ed by E. Inönü and M. Serdaroglu.

[18] M. Günaydin and S.J. Hyun, J. Math. Phys. 29 (1988) 2367.

[19] M. Günaydin, Nucl. Phys. B 6 (Proc. Suppl.) (1989) p. 140.

[20] M. Günaydin, D. Minic and M. Zagermann, hep-th/9806042, Nucl. Phys. B534(1998) 96-120.

[21] M. Günaydin, D. Minic and M. Zagermann, hep-th/9810226, Nucl. Phys. B544 (1999) 737-758.

[22] M. Günaydin and S. Takemae, "Unitary Supermultiplets of $O S p\left(8^{*} \mid 4\right)$ and the $A d S_{7} / C F T_{6}$ Duality", hep-th/9910110.

[23] M. Günaydin, G. Sierra and P.K. Townsend, Nucl. Phys. B 274 (1986) 429 . 
[24] M. Günaydin, in Proceedings of the Trieste conference "Supermembranes and Physics in 2+1 dimensions", eds. M. J. Duff, C. N. Pope and E. Sezgin, World Scientific, 1990, pp.442.

[25] M. Günaydin and R. Scalise, Jour. Math. Phys. 32 (1991) 599.

[26] M. Günaydin, J. Math. Phys. 29 (1988) 1275.

[27] P.Claus, M. Gunaydin, R. Kallosh, J. Rahmfeld and Y. Zunger, JHEP 9905 (1999) 019, hep-th/9905112; P. Claus, R. Kallosh and J. Rahmfeld, hep-th/9906195.

[28] M. Günaydin, Nuovo Cimento 29A (1975) 467.

[29] M. Günaydin, Ann. Israel Physical Society 3 (1980) 279.

[30] M. Günaydin, "Generalized conformal and superconformal group actions and Jordan algebras," Mod. Phys. Lett. A8, 1407 (1993) hepth/9301050].

[31] M. Günaydin, in "Elementary Particles and the Universe: Essays in Honor of Murray Gell-Mann", ed. by J.H. Schwarz ( Cambridge University Press, 1991).

[32] G. Mack and A. Salam, Ann. Phys. 53 (1969) 174; G. Mack, Comm. Math. Phys. 55 (1977) 1.

[33] M. Gunaydin, "AdS/CFT Dualities and the Unitary Representations of Non-compact Groups and Supergroups: Wigner versus Dirac", invited talk at the VIth International Wigner Symposium ( Aug. 1999, Istanbul) , hep-th/0008063.

[34] M. Gunaydin, in preparation

[35] M. Günaydin, K. Koepsell and H. Nicolai, "Conformal and Quasiconformal Realizations of Exceptional Lie Groups", hep-th/0008063.

[36] S. Fernando, M. Gunaydin and S. Takemae, in preparation. 
[37] L. Andrianopoli, S. Ferrara, E. Sokatchev and B. Zupnik, hepth/9912007 ; Ferrara and E. Sokatchev, hep-th/9912168; S. Ferrara and E. Sokatchev, hep-th/0001178; hep-th/0003051; P. Heslop and P. Howe, hep-th/0009217; P. Heslop and P. Howe, Class. Quant. Grav. 17 (2000) 3743 hep-th/0005135; P. S. Howe, hep-th/0008048.

[38] J. W. van Holten and A. van Proeyen, "N=1 Supersymmetry Algebras In $\mathrm{D}=2, \mathrm{D}=3, \mathrm{D}=4$ Mod-8," J. Phys. A A15, 3763 (1982).

[39] P. Horava and E. Witten, " Heterotic and Type I String dynamics from eleven dimensions", Nucl. Phys. B460 (1996) 506 (hep-th/9510209).

[40] P. Horava, "M-theory as a holographic field theory," Phys. Rev. D59, 046004 (1999) hep-th/9712130. 